\title{
Ensinando o pai a brincar com seu bebê com síndrome de Down
}

\section{Teaching the father how to play with his baby with Down's syndrome}

\author{
Nancy Capretz Batista da Silva ${ }^{1}$ \\ Ana Lúcia Rossito Aiello
}

\begin{abstract}
RESUMO
Os membros da família de uma pessoa com necessidades educacionais especiais podem precisar de assistência e deveriam ser clientes de serviços e intervenções profissionais. Embora as mães sejam o principal cuidador da criança em contato com os programas de intervenção precoce, estudos têm cada vez mais mostrado a importância do pai e de sua participação. Este estudo ${ }^{3}$ teve como objetivo avaliar se uma intervenção direcionada ao pai altera seus padrões de interações diádicas com seu bebê com síndrome de Down em situações de brincadeira. Foi utilizado o Protocolo de Categorias de Análise das Filmagens de Interação na análise de cinco filmagens de interação: duas antes, uma durante, uma após a intervenção e uma de follow-up. O pai participou de treino de comportamentos para o desenvolvimento do bebê, com orientações sobre como estimular a criança. Houve o aumento na frequência de comportamentos positivos após o feedback fornecido aos pais e ao longo da intervenção até o final desta, indicando um efeito positivo das orientações fornecidas. As mães, que não passaram pelo treino, não tiveram os mesmos ganhos. Contudo, os efeitos nos pais não se mantiveram no follow-up. As crianças também passaram a exibir mais comportamentos positivos em interação. Conclui-se que os objetivos foram alcançados e que é possível envolver o pai nos
\end{abstract}

${ }^{1}$ Doutora em Educação Especial pela Universidade Federal de São Carlos, Brasil. E-mail: nancycbs@gmail.com.

${ }^{2}$ Doutora em Psicologia Experimental pela Universidade de São Paulo. Professora Adjunta da Universidade Federal de São Carlos, Brasil. E-mail: ana.aiello@terra.com.br.

${ }^{3}$ Pesquisa financiada pela CAPES (Coordenação de Aperfeiçoamento de Pessoal de Nível Superior), Brasil. 
programas de intervenção precoce visando ao melhor desenvolvimento de bebês com atraso.

Palavras-chave: intervenção precoce; estimulação precoce; bebê com sindrome de Down; interação pai-bebê.

\begin{abstract}
Family members of a person with special educational needs may need assistance and should be customers of professional services and interventions. Although mothers are the child primary caregiver in touch with early intervention programs, studies have increasingly shown the importance of fathers and their participation. This study aimed to evaluate whether an intervention directed at fathers changes their patterns of dyadic interactions with their baby with Down syndrome in play situations. It was used the Protocol of Analysis Categories of Interaction Footages in the analysis of five interaction shots: two before, one during, one after the intervention and a follow-up one. The father attended the behaviors training to the baby's development, with guidance on how to stimulate the child. There was an increase in the frequency of positive behaviors after the feedback given to parents and during the intervention by the end of this, indicating a positive effect of the guidelines provided. Mothers, who have not undergone the training, did not have the same gains. However, the effects on fathers were not sustained at follow-up. Children have also started to exhibit more positive behaviors in interaction. It is concluded that the objectives were achieved and it is possible to involve the father in early intervention programs aimed at better development of babies with developmental delay.
\end{abstract}

Keywords: early intervention; early stimulation; baby with Down's syndrome; father-baby interaction.

\title{
Introdução
}

A grande maioria das crianças experiencia com a família "as primeiras situações de aprendizagem e introjeção de padrões, normas e valores, e se a família não estiver funcionando adequadamente, as interações, principalmente pais-bebê e com a sociedade, serão prejudicadas" (COLNAGO, 2000, p. 161).

Desta forma, quando há na família um bebê com atraso no desenvolvimento, é importante que seja dado suporte formal e informal a essa família para que sejam capazes de lidar com as demandas do dia a dia de forma eficaz 
e para que estimulem o bebê satisfatoriamente, preservando o cumprimento das necessidades individuais. Neste sentido, o nascimento de uma criança com deficiência causa impactos individuais, conjugais e parentais diferentes em mães e pais (PELCHAT; LEFEBVRE; LEVERT, 2007), ressaltando a importância de suportes práticos e emocionais (CARPENTER, 2000), considerando também as peculiaridades dos indivíduos.

Além disso, quando a criança é pequena, os pais são o principal foco de atenção do profissional e existe um reconhecimento cada vez maior que os membros da família de uma pessoa com necessidades educacionais especiais possam precisar de assistência e deveriam ser clientes de serviços e intervenções profissionais (GALLAGHER, 1990). De acordo com Omote (1996), a família da pessoa com deficiência tem sido reconhecida como importante alvo de estudo e de atenção profissional para compreender suas dificuldades e limitações e educá-la adequadamente, mas também para suprir suas "necessidades especiais de atendimento, decorrentes da sua condição de ser mãe, pai, irmão ou irmã de um indivíduo reconhecido e tratado como deficiente" (p. 516).

Segundo Bolsanello (2003), a legislação brasileira considera a estimulação precoce como o primeiro programa de atendimento destinado principalmente à crianças de alto risco ou possuidoras de deficiências (auditiva, física, intelectual, visual, múltipla, atrasos no desenvolvimento), na faixa etária de zero a três anos. Os primeiros anos de vida são críticos para o desenvolvimento da criança, justificando a implementação de programas de intervenção precoce, cujos princípios fundamentais são: serviços centrados na família, focalizar competências e capacidade de recuperação da criança e da família, considerar o sistema social, ocorrer em nível de interações e relações familiares e ser empiricamente orientado (PEREIRA-SILVA; DESSEN, 2005).

Desta forma, Williams e Aiello (2004) enfatizam a necessidade de enfocar a intervenção na família e não apenas na criança, enquanto Kumpfer e Alvarado (2003) ressaltam que intervenções focadas na família aumentam a efetividade dos programas. Os benefícios da participação das famílias nos programas de intervenção precoce seriam: melhor adaptação ao problema da criança, bem-estar no relacionamento conjugal e nos papéis parentais e percepções e emoções mais positivas sobre vários aspectos da vida (PELCHAT; LEFEBVRE; LEVERT, 2007).

De acordo com Spiker e Hopmann (1997), acredita-se que a relação pais-criança seja crítica para o desenvolvimento da criança. Assistir às famílias para começara a estabelecer interações pais-criança agradáveis e estimulantes, fator essencial para facilitar todo seu desenvolvimento futuro, constitui objetivo apropriado e importante da intervenção precoce. Com relação à criança com Síndrome de Down, embora muitas vezes ela seja o foco de atenção, os membros 
da família e outros cuidadores também podem precisar de intervenção direta para se tornarem competentes fontes de informação para outros e agentes para intervenções terapêuticas. Kumpfer e Alvarado (2003) sugerem ações para esse tipo de intervenção ser efetiva, como incluir estratégias para melhorar relações familiares e utilizar métodos interativos de treino de habilidades.

Embora as mães sejam o principal cuidador da criança em contato com os programas de intervenção, estudos nos últimos 40 anos têm cada vez mais mostrado a importância do pai e de sua participação em programas de intervenção precoce. Em relação a isso, Colnago afirma que é necessário:

[...] delinear modelos de programas de intervenção e de implementação de serviços em função das características individuais e locais das famílias de deficientes, principalmente no que tange às informações advindas do pai e dos irmãos, porque mesmo nos estudos que abordam algumas questões acerca do pai e dos irmãos, as informações foram obtidas através das percepções das mães. (COLNAGO, 2000, p. 162).

Ainda que haja consenso na literatura sobre desenvolvimento humano a respeito da importância do envolvimento positivo do pai (LAMB, 1997; FLOURI; BUCHANAN, 2003; BOECHLER et al., 2003; HARRISON; MAGILL-EVANS, 2003; CIA et al., 2004; NUNES et al.; 2008; DA SILVA; AIELLO, 2009), muitos serviços são dirigidos às mães e seus filhos. A maioria da literatura sobre os serviços sociais e de saúde, por exemplo, foca em mães e crianças (ENGLE; BREAUX, 1998).

As razões para a inclusão apenas da mãe nos programas de atendimento às crianças com deficiências englobam sua maior disponibilidade para interação, desconforto dos profissionais dos programas (a maioria mulheres) para lidar com homens e despreparo dos educadores, os quais já não são treinados para trabalhar com famílias, ainda menos para trabalhar com o pai (TURBIVILLE; MARQUIS, 2001). No Brasil, as experiências de inclusão do pai nos programas de intervenção precoce para crianças com deficiência são mais escassas que em alguns países, como os Estados Unidos.

Colnago (2000) elaborou e testou um programa de intervenção para pais de crianças com Síndrome de Down em idade entre um mês e quinze meses, no interior do Estado de São Paulo, procurando mostrar aos pais a importância de sua participação ativa no processo de desenvolvimento da criança, dando-lhes suporte informativo e emocional por meio de orientações em grupo. As análises 
mostraram que, à medida que os pais aprendiam sobre o desenvolvimento da criança e as formas de cuidá-la, ocorria a percepção de que o filho estava evoluindo, atingindo os marcos do desenvolvimento, e o estresse parental diminuía.

Uma intervenção breve com pais de crianças com Síndrome de Down, em duas cidades do interior do Estado de São Paulo, foi realizada no estudo de Da Silva (2007) a partir da avaliação do envolvimento e da interação do pai com seu filho. Metade dos pais passava boa parte do dia com suas crianças. Nas interações, "olhar na direção da criança", foi o comportamento positivo de maior frequência para os pais; nenhum genitor corrigiu comportamentos inadequados; e o comportamento negativo de maior frequência foi "comentários negativos". O comportamento positivo mais emitido pelas crianças foi "prestar atenção" e o comportamento negativo foi "desobediência". Ainda que tenham sido fornecidas informações, os efeitos dessa intervenção nos participantes não foram avaliados.

Entre os programas voltados para o pai descritos na literatura, a maioria tem o envolvimento como alvo, procurando aprimorar habilidades parentais, melhorar a interação, prover suporte aos pais e discutir questões sobre emprego (McBRIDE, 1990; ENGLE; BREAUX, 1998; CABRERA et al., 2002; MINCY; POUNCY, 2002; RAIKES et al., 2002; BARKER et al., 2004; BARLLET; VANN, 2004; McALLISTER; WILSON; BURTON, 2004; DOHERTY; ERICKSON; LA ROSSA, 2006; MAGGIL-EVANS et al., 2006; CIA, 2009). Mas eles ainda são poucos e carecem de avaliação dos efeitos de forma sistemática, além de não avaliarem os efeitos nos filhos. Quando se trata de crianças com necessidades educacionais especiais, a escassez de estudos na área de intervenção precoce que inclua uma figura paterna é ainda maior.

Por último, é importante que os profissionais reconheçam que a maneira como o pai mais se engaja com seus filhos é brincando (FAGAN; IGLESIAS, 1999; CALDERA, 2004; QUESENBERRY; OSTROSKY; CORSO, 2007). Bruner (1986) acredita que o ato de brincar apresenta condições ótimas para explorar e desenvolver habilidades mais complexas.

Da Silva, Oliveira e Sás (2008) sugerem o uso de brincadeiras e jogos no ensino do indivíduo com necessidades educacionais especiais ao reunirem evidências sobre a importância do brincar para o desenvolvimento. Mas é necessário considerar que alguns fatores influenciam o desempenho da criança no brincar, como o ambiente no qual ela se encontra, suas características individuais e o vínculo com a pessoa que aplica as atividades (EMMEL, 2004).

Diante do exposto, o objetivo deste estudo foi avaliar se uma intervenção direcionada ao pai altera seus padrões de interações diádicas com seu bebê com síndrome de Down em situações de brincadeira. 


\section{Método}

Este estudo foi aprovado pelo Comitê de Ética em Pesquisa da UFSCar (CAAE 0030.0.135.000-08, Parecer no. 278/2008). Os participantes assinaram um Termo de Consentimento Livre e Esclarecido (TCLE).

\section{Participantes}

Participaram deste estudo cinco famílias (casais heterossexuais com filhos) de bebês com Síndrome de Down, residentes em duas cidades do interior do Estado de São Paulo (1-3 em São Carlos e 4-5 em Ribeirão Preto).

A Tabela 1 apresenta a caracterização demográfica das famílias participantes. Podemos observar que todos os bebês frequentavam serviços públicos de atendimento (serviços de fisioterapia - instituição A, terapia ocupacional instituição B e estimulação infantil - instituição C). A idade média dos pais foi de 41 anos (Desvio Padrão = 6,47), para as mães foi de 37 anos (Desvio Padrão $=3,97$ ) e para os bebês foi de 15 meses (Desvio Padrão $=3,01$ ).

TABELA 1 - CARACTERIZAÇÃO DEMOGRÁFICA DAS FAMÍLIAS PARTICIPANTES

\begin{tabular}{|c|c|c|c|c|c|c|c|c|c|c|}
\hline \multirow[b]{2}{*}{ 丞 } & & \multicolumn{2}{|c|}{ CRIANÇA } & \multicolumn{3}{|c|}{ PAI } & \multicolumn{4}{|c|}{ MÃE } \\
\hline & Filhos & $\begin{array}{l}\text { Idade* }^{*} \\
\text { (meses) }\end{array}$ & $\begin{array}{l}\text { Insti- } \\
\text { tuição }\end{array}$ & $\begin{array}{l}\text { Idade* } \\
\text { (anos) }\end{array}$ & Profissão & $\begin{array}{l}\text { Escola- } \\
\text { ridade }\end{array}$ & $\begin{array}{l}\text { Idade* } \\
\text { (anos) }\end{array}$ & Profissão & $\begin{array}{l}\text { Escola- } \\
\text { ridade }\end{array}$ & $\begin{array}{c}\text { Renda per } \\
\text { capita }^{1}\end{array}$ \\
\hline 1 & 1 & 14 & $\mathrm{~A}$ & 36 & Músico & $\begin{array}{l}\text { Superior } \\
\text { Com- } \\
\text { pleto }\end{array}$ & 35 & $\begin{array}{l}\text { Professora } \\
\text { universi- } \\
\text { tária }\end{array}$ & $\begin{array}{l}\text { Superior } \\
\text { Com- } \\
\text { pleto }\end{array}$ & $\mathrm{R} \$ 1500,00$ \\
\hline 2 & 1 & 19 & A & 41 & Soldador & $\begin{array}{l}\text { Funda- } \\
\text { mental } \\
\text { Incomp. }\end{array}$ & 40 & Do lar & $\begin{array}{l}\text { Médio } \\
\text { Incomp. }\end{array}$ & $\mathrm{R} \$ 400,00$ \\
\hline 3 & $4^{2}$ & 13 & B & 32 & $\begin{array}{l}\text { Líder de } \\
\text { produção }\end{array}$ & $\begin{array}{l}\text { Médio } \\
\text { Com- } \\
\text { pleto }\end{array}$ & 30 & $\begin{array}{l}\text { Operária } \\
\text { de fábrica }\end{array}$ & $\begin{array}{l}\text { Médio } \\
\text { Com- } \\
\text { pleto }\end{array}$ & $\begin{array}{l}\mathrm{R} \$ \\
767,00\end{array}$ \\
\hline 4 & 2 & 12 & $\mathrm{C}$ & 51 & Lustrador & $\begin{array}{l}\text { Médio } \\
\text { Incomp. }\end{array}$ & 38 & Do lar & $\begin{array}{l}\text { Médio } \\
\text { Com- } \\
\text { pleto }\end{array}$ & $\mathrm{R} \$ 253,75$ \\
\hline 5 & 2 & 19 & $\mathrm{C}$ & 43 & $\begin{array}{l}\text { Operador } \\
\text { de fábrica }\end{array}$ & $\begin{array}{l}\text { Médio } \\
\text { Com- } \\
\text { pleto }\end{array}$ & 41 & Diarista & $\begin{array}{l}\text { Médio } \\
\text { Com- } \\
\text { pleto }\end{array}$ & $\mathrm{R} \$ 312,50$ \\
\hline
\end{tabular}

*No momento do início da participação da família no estudo.

${ }^{1}$ Salário mínimo de junho de 2008 a fevereiro de 2009: R \$ 415,00.

${ }^{2}$ Apesar de haver mais 3 filhos menores de idade, apenas a criança alvo do estudo residia com esta família. 
Local

Os encontros semanais foram realizados nas residências das famílias, em um espaço comum, como sala, cozinha ou varanda, com duração de aproximadamente uma hora e meia.

\section{Materiais e equipamentos}

Foram necessários para a consecução do estudo: (a) câmera digital modelo Sony DSC Cyber Shot W120 para filmar as interações dos pais com as crianças; (b) solicitação às instituições para recrutamento das famílias; (c) brinquedos para estruturar situação de interação; e (d) microcomputador para observação e análise das interações. Os brinquedos estavam de acordo com a faixa etária das crianças e eram disponíveis comercialmente (grupo 1: livro de figuras com rótulos, carrinho, boneca e telefone; grupo 2: pandeiro, urso de pelúcia, casinha de encaixe de formas e fantoche).

Para a análise das interações, foi utilizado o Protocolo de Categorias de Análise das Filmagens de Interação (BUONADIO, 2003). Ele contém quatorze categorias de comportamentos dos pais - comentário positivo, olhar dirigido à criança, dar dicas (verbais ou gestuais), ajuda física, comentários negativos, imitação da vocalização da criança e/ou expansão, correção de comportamento inadequado, repressão física, fazendo uma questão, modelando, rotulando, comando, contato físico positivo e brincar sozinho - e oito categorias de comportamentos da criança - prestando atenção, obediência, desobediência, vocalizações positivas, vocalizações negativas, comportamento físico negativo, fazendo uma questão e brincar sozinho. Duas categorias acrescentadas ao protocolo no estudo de Da Silva (2007) foram utilizadas: motivar a criança para a tarefa e criança engajada na tarefa. Os dados foram avaliados de acordo com procedimento de registro por amostragem de tempo (10 segundos de observação e 10 segundos de registro). Este protocolo possibilita uma análise funcional dos comportamentos dos familiares em interação, sendo que todas as categorias possuem definições operacionais.

\section{Procedimentos}

As famílias foram contatadas por meio dos serviços que recebiam (conforme Tabela 1). Após algumas visitas para coleta inicial de dados, foram realizadas duas filmagens em dias diferentes das interações pai-bebê e mãe-bebê em situações de brincadeira, com duração de 10 minutos para cada díade (as 
análises para a tríade não serão descritas aqui). No total eram 30 minutos de filmagem por dia, iniciando sempre pela díade pai-bebê. Ainda foram realizadas três filmagens: durante a intervenção, na finalização da mesma e após 6-7 meses. O grupo 1 de brinquedos era fornecido primeiramente na interação, intercalando a ordem com o grupo 2. Na segunda filmagem, ocorria a inversão da ordem de apresentação dos grupos de brinquedos.

Foi solicitado que o pai e a mãe brincassem com seu filho/ ou sua filha, como faziam normalmente quando estavam juntos. Ao genitor que não estivesse participando era solicitado que se retirasse do local, evitando eventuais interferências na díade observada. Durante as filmagens, a pesquisadora tinha uma postura apenas de observação, não sendo encorajada nenhuma interação verbal ou comportamental. As interações foram avaliadas com o protocolo descrito anteriormente e as famílias sempre recebiam uma cópia das filmagens.

Foi fornecido um feedback sobre as duas filmagens iniciais para pai e mãe. Em seguida, durante a intervenção (ver detalhes em Da Silva, 2011), estabeleceu-se treino semanal somente com o pai para instalar novos comportamentos no repertório da criança para o seu melhor desenvolvimento, os quais consistiam em itens do Inventário Portage Operacionalizado (WILLIAMS; AIELLO, 2001). As sessões de treino tiveram apoio de textos sobre assuntos levantados pelos próprios pais e pela literatura como importantes (por exemplo, desenvolvimento das crianças com síndrome de Down, legislação, manejo de comportamento etc.).

O pai era ensinado por modelo e instruções diretas a preparar o ambiente; dar instruções mais claras e objetivas; rotular ações, objetos e pessoas e suas características; reforçar diferencialmente diferentes níveis de desempenho da criança em diferentes estágios do treino; usar diferentes níveis de ajuda (modelo, ajuda física, dicas verbais e gestuais); retirada gradual de ajuda; redirecionamento de comportamentos errados ou inadequados; ignorar comportamento inconsequente e reforçar seletivamente outro comportamento apropriado.

As famílias não receberam feedback sobre as interações filmadas durante a intervenção, apenas no final da mesma e seis a sete meses depois, após nova observação no follow-up.

\section{Resultados}

A Tabela 2 apresenta os resultados das categorias de comportamentos dos pais e das mães em interações diádicas. 
DA SILVA, N. C. B.; AIELLO, A. L. R. Ensinando o pai a brincar com seu bebê...

TABELA 2 - COMPORTAMENTOS DOS GENITORES EM DÍADES COM OS BEBÊS

\begin{tabular}{|c|c|c|c|c|c|c|c|c|c|c|c|c|}
\hline \multirow{2}{*}{ Cptos. } & \multicolumn{2}{|c|}{ Pré-teste 1} & \multicolumn{2}{|c|}{ Pré-teste 2} & \multicolumn{2}{|c|}{ Sondagem } & \multicolumn{2}{|c|}{ Pós-teste } & \multicolumn{2}{|c|}{ Follow-up } & \multicolumn{2}{|c|}{ Total } \\
\hline & Pai & Mãe & Pai & Mãe & Pai & Mãe & Pai & Mãe & Pai & Mãe & Pai & Mãe \\
\hline 1 & 18 & 30 & 13 & 25 & 26 & 26 & 11 & 37 & 3 & 29 & 71 & 147 \\
\hline 2 & 52 & 79 & 68 & 74 & 57 & 63 & 28 & 58 & 19 & 19 & 224 & 293 \\
\hline 3 & 149 & 149 & 148 & 147 & 143 & 147 & 148 & 149 & 149 & 149 & 737 & 741 \\
\hline 4 & 5 & 7 & 3 & 13 & 13 & 28 & 11 & 17 & 14 & 28 & 46 & 93 \\
\hline 5 & 4 & 7 & 8 & 6 & 10 & 22 & 9 & 12 & 4 & 17 & 35 & 64 \\
\hline 6 & 7 & 9 & 18 & 24 & 20 & 23 & 8 & 23 & 0 & 13 & 53 & 92 \\
\hline 7 & 7 & 6 & 6 & 7 & 10 & 4 & 18 & 14 & 13 & 5 & 54 & 36 \\
\hline 8 & 0 & 0 & 0 & 0 & 0 & 0 & 0 & 0 & 0 & 0 & 0 & 0 \\
\hline 9 & 27 & 19 & 10 & 26 & 19 & 35 & 35 & 35 & 45 & 53 & 136 & 168 \\
\hline 10 & 18 & 16 & 13 & 29 & 27 & 15 & 29 & 27 & 17 & 29 & 104 & 116 \\
\hline 11 & 20 & 22 & 15 & 19 & 32 & 22 & 23 & 21 & 47 & 25 & 137 & 109 \\
\hline 12 & 21 & 17 & 19 & 12 & 20 & 12 & 8 & 8 & 7 & 4 & 75 & 53 \\
\hline 13 & 2 & 14 & 0 & 2 & 18 & 9 & 4 & 14 & 8 & 11 & 32 & 50 \\
\hline 14 & 3 & 5 & 0 & 0 & 0 & 1 & 0 & 0 & 8 & 1 & 11 & 7 \\
\hline 15 & 0 & 0 & 2 & 0 & 0 & 0 & 0 & 0 & 1 & 0 & 3 & 0 \\
\hline 16 & 32 & 59 & 32 & 46 & 38 & 44 & 39 & 46 & 40 & 53 & 181 & 248 \\
\hline Total & 365 & 439 & 355 & 430 & 433 & 451 & 371 & 461 & 375 & 436 & 1899 & 2217 \\
\hline
\end{tabular}

Cptos: comportamentos; 1: comentários positivos; 2: motivar a criança para a tarefa; 3 : olhar na direção da criança; 4: dar dicas verbais; 5: dar dicas gestuais; 6: dar ajuda física; 7: imitação ou expansão da vocalização da criança; 8: correção de comportamento inadequado; 9: fazer questão; 10: modelando; 11: rotulando; 12: contato físico positivo; 13: comentários negativos; 14: repressão física; 15: brincar sozinho; 16: dar comando.

Os comportamentos que os genitores mais emitiram durante as interações foram olhar para a criança, motivar para a tarefa e dar comandos. Outros comportamentos positivos ocorreram em baixa frequência, como imitar ou expandir a vocalização da criança, dicas verbais e dicas gestuais, porém os comportamentos com menor frequência foram negativos, como repressão física e brincar sozinho. Não houve correção para o comportamento inadequado em nenhuma interação diádica.

De forma geral, em díade com os filhos, as mães fizeram mais comentários positivos, motivaram mais as crianças para a tarefa, olharam mais em direção da criança, deram mais dicas verbais e gestuais, forneceram mais ajuda física, fizeram mais questões, deram mais modelos, fizeram mais comentários negativos e deram mais comandos que os pais. Os pais, por sua vez, imitaram ou expandiram mais as vocalizações das crianças, rotularam mais vezes, tiveram mais contato físico positivo com as crianças e realizaram mais repressão física. 
Após o feedback aos pais sobre seus comportamentos, nas interações diádicas, durante a intervenção, a frequência de vários comportamentos positivos do pai aumentou, como comentários positivos, dicas verbais, dicas gestuais, ajuda física, imitação/expansão da vocalização da criança, modelar, rotular e dar comando. Além disso, não houve mais nenhum registro de repressão física. Para as mães, apenas dicas verbais, dicas gestuais e fazer questão aumentaram em frequência. Por outro lado, os pais fizeram mais comentários negativos e olharam menos para a criança.

Ao final da intervenção, foi observado que comportamentos do pai, como imitar/expandir a vocalização da criança, fazer questão, modelar e dar comando ocorreram ainda mais. Olhar para a criança apresentou a mesma frequência inicial e a ocorrência de comentários negativos diminuiu. Contudo, outros comportamentos, como comentários positivos, motivar a criança para a tarefa, dicas verbais, dicas gestuais, ajuda física, rotular e contato físico positivo diminuíram em frequência.

Para as mães, surgiram mais comentários positivos e imitação/expansão da vocalização da criança. Porém, comportamentos como motivar a criança para a tarefa, dicas verbais, dicas gestuais e contato físico positivo diminuíram.

Entre seis a sete meses após o término da intervenção, observou-se para os pais que comentários positivos, motivar para a tarefa, dicas gestuais, ajuda física, imitar/expandir a vocalização da criança, modelar e contato físico positivo diminuíram em frequência. Por outro lado, dicas verbais, fazer questão, rotular e dar comando ocorreram mais frequentemente.

Para as mães, comentários positivos, motivar para a tarefa, ajuda física, imitar/expandir a vocalização da criança e contato físico positivo também diminuíram em frequência. Todavia, dicas verbais, dicas gestuais, fazer questão, modelar, rotular e dar comando ocorreram em maior frequência.

A Tabela 3 apresenta os resultados das categorias de comportamentos dos bebês em interações diádicas com pais e mães.

As crianças emitiram mais prestar atenção, se engajar na tarefa e vocalizações positivas nas interações. Obedecer apresentou uma baixa frequência, contudo, foi superior à frequência de desobediência. As crianças não fizeram questões e brincar sozinho foi o comportamento não favorecedor de interações que ocorreu em maior frequência.

Ao longo das fases do estudo, a frequência de comportamentos das crianças interagindo com seus genitores aumentou gradativamente. As crianças apresentaram uma maior frequência de comportamentos em interação com a mãe e vocalizaram mais (positivamente) com o pai. 
TABELA 3 - COMPORTAMENTOS DOS BEBÊS EM DÍADES COM OS GENITORES.

\begin{tabular}{|c|c|c|c|c|c|c|c|c|c|c|c|c|}
\hline \multirow{2}{*}{ Cptos. } & \multicolumn{2}{|c|}{ Pré-teste 1} & \multicolumn{2}{|c|}{ Pré-teste 2} & \multicolumn{2}{|c|}{ Sondagem } & \multicolumn{2}{|c|}{ Pós-teste } & \multicolumn{2}{|c|}{ Follow-up } & \multicolumn{2}{|c|}{ Total } \\
\hline & Pai & Mãe & Pai & Mãe & Pai & Mãe & Pai & Mãe & Pai & Mãe & Pai & Mãe \\
\hline 1 & 115 & 124 & 112 & 116 & 125 & 135 & 117 & 138 & 118 & 130 & 587 & 643 \\
\hline 2 & 11 & 19 & 8 & 8 & 8 & 7 & 12 & 6 & 10 & 15 & 49 & 55 \\
\hline 3 & 24 & 7 & 23 & 32 & 33 & 25 & 51 & 46 & 53 & 47 & 184 & 157 \\
\hline 4 & 60 & 75 & 76 & 79 & 97 & 79 & 104 & 111 & 95 & 111 & 432 & 455 \\
\hline 5 & 0 & 0 & 0 & 0 & 0 & 0 & 0 & 0 & 0 & 0 & 0 & 0 \\
\hline 6 & 1 & 6 & 2 & 3 & 3 & 4 & 1 & 1 & 0 & 1 & 7 & 15 \\
\hline 7 & 1 & 8 & 3 & 9 & 1 & 7 & 4 & 3 & 6 & 3 & 15 & 30 \\
\hline 8 & 2 & 13 & 7 & 4 & 1 & 4 & 3 & 3 & 0 & 4 & 13 & 28 \\
\hline 9 & 24 & 6 & 23 & 18 & 12 & 18 & 7 & 2 & 24 & 11 & 90 & 55 \\
\hline Total & 238 & 258 & 254 & 269 & 280 & 279 & 299 & 310 & 306 & 322 & 1377 & 1438 \\
\hline
\end{tabular}

Cptos: comportamentos; 1: prestar atenção; 2: obediência; 3: vocalização positiva; 4: engajada na tarefa; 5: fazendo questão; 6: desobediência; 7: vocalização negativa; 8: comportamento físico negativo; 9: brincar sozinho.

\section{Discussões}

Deve-se considerar que, no dia a dia, as brincadeiras podem acontecer em situações de cuidados das crianças e de rotinas das famílias, como na hora do banho, na hora de comer, de ir para a cama, esperar no consultório médico, no caminho até a casa dos avós ou até algum atendimento, no parque, etc. Por isso, por mais que se tenha criado uma situação para padronizar as observações e que representasse algo mais interessante para os homens, a situação pode não ser a situação normal de brincadeira das famílias.

Há também possível alteração do comportamento dos participantes devido à presença da pesquisadora como observador. Contudo, de acordo com Dessen (1996), o grau de intimidade é intensificado quando o delineamento de pesquisa é longitudinal e a coleta de dados efetuada na residência das famílias, por um único pesquisador.

No geral, os pais deste estudo apresentaram o mesmo padrão comportamental encontrado no estudo de Da Silva (2007) com pais de crianças com síndrome de Down. Na presente investigação, o aumento na frequência de alguns comportamentos positivos após o feedback fornecido aos pais indica um efeito positivo das orientações fornecidas.

No mesmo sentido, observou-se que os efeitos não foram os mesmos em ambos os genitores, sendo que foram registradas mudanças em mais compor- 
tamentos dos pais do que das mães, o que pode ser atribuído à participação dos mesmos no treino oferecido. Como as mães já apresentavam mais comportamentos positivos com os bebês nas interações, foi muito importante o aumento da frequência de comportamentos positivos dos pais após o feedback, no sentido de haver maior coerência no nível de estimulação proporcionado por ambos os genitores aos bebês.

A ausência de correção para o comportamento inadequado observado, não significa que as crianças não tenham se comportado inadequadamente em alguns momentos. Essa ausência pode estar relacionada à possível consideração dos pais da situação de filmagem como imprópria para colocar o comportamento inadequado da criança em extinção ou time-out, uma vez que essas técnicas reduziriam o tempo de interação entre genitores e crianças.

Foi interessante notar que, diante dos diferentes padrões de interação de mães e pais, os bebês apresentaram uma maior frequência de comportamentos em interação com a mãe e vocalizaram mais (positivamente) com o pai, o qual imitava ou expandia mais suas vocalizações; o que demonstra a reciprocidade no comportamento daqueles que interagem.

A diminuição na frequência de comportamentos positivos dos pais após o término da intervenção, sugere que um feedback mais direto sobre os comportamentos observados pode ser mais eficiente que um treino de comportamentos em outra situação de ensino e/ou que a intervenção é menos eficaz com pais quando ofertada em longo prazo, perdendo seu poder de modificação e manutenção de comportamentos.

Ainda ao final das intervenções, notou-se que as mães não tiveram aumento na frequência no mesmo número de comportamentos que os pais. Mais uma vez, a intervenção parece causar mudanças no repertório de interação dos pais que não são observadas com a mesma magnitude no comportamento das mães.

Por fim, no follow-up, os genitores passaram a responder a uma nova fase no desenvolvimento das crianças, com aumento na frequência de comportamentos que as ajudavam a emitir as primeiras palavras ou frases, ainda que a intervenção não tenha mantido a maior frequência de todos os comportamentos positivos desejáveis.

O gradual aumento na frequência de comportamentos dos bebês pode estar relacionado ao desenvolvimento de cada vez mais habilidades, conforme esperado com o treino do pai. Na mesma direção, o aumento na frequência de comportamentos positivos do pai após o feedback pode ter ocorrido porque as crianças respondiam cada vez mais prontamente. Ou, por outro lado, as crianças desenvolveram novos repertórios em interação em resposta ao aumento na frequência de comportamentos positivos dos pais. 


\section{Conclusões}

Conclui-se que o objetivo deste estudo, avaliar se uma intervenção direcionada ao pai altera seus padrões de interações com seu bebê com síndrome de Down em situações de brincadeira, foi alcançado. Embora o repertório comportamental dos bebês não tenha sido relatado mais minuciosamente na presente investigação, foi possível observar aumento na frequência de comportamentos dos mesmos em conformidade com o aumento na frequência de comportamentos dos pais.

Assim, diante da importância do brincar para o desenvolvimento infantil e do interesse dos pais em interagir com seus filhos por este meio, é possível envolver o pai nos programas de intervenção precoce com objetivos de ganho não só para os bebês com atrasos no desenvolvimento, como também para a família como um todo, a qual pode adquirir novas habilidades e ter suas necessidades atendidas.

\section{REFERÊNCIAS}

BARKER, G.; NASCIMENTO, M.; SEGUNDO, M.; PULERWITZ, J. How do we know if men have changed? Promoting and measuring attitude change with young men: Lessons from Program H in Latin America. Brasília, 2004. Disponível em: <http://www. promundo.org.br/areas-de-atuacao/areas-de-atuacao-posts/materiais-produzidos-pelo-programa-h/>. Acesso em: 15/07/2007.

BARLLET, D.; VANN, N. Review of the state of practical work. In: BARKER, G.; BARTLLET, D.; BEARDSHAW, T.; BROWN, J.; BURGESS, A.; LAMB, M.; LEWIS, C.; RUSSELL, G.; VANN, N. Early Childhood Development: Practice and Reflections - Supporting fathers: Contributions from the International Fatherhood Summit 2003. Oxford: Bernard Van Leer Foundation, p. 78, 2004.

BOECHLER, V.; HARRISON, M. J.; MAGILL-EVANS, J. Father-child teaching interactions: The relationship to father involvement in caregiving. Journal of Pediatric Nursing, v. 18, n. 1, p. 46-51, fev. 2003.

BOLSANELLO, M. A. Concepções sobre os procedimentos de intervenção e avaliação de profissionais em estimulação precoce. Educar em Revista, Curitiba, v. 22, p. 343$355,2003$.

BRUNER, J. S. Juego, pensamiento y lenguaje. Perspectivas, UNESCO, v. 16, n. 1, p. 79-86, 1986. 
BUONADIO, M. C. Análise da interação de mães com deficiência mental e seus filhos: intervenção domiciliar. Dissertação (Mestrado em Educação Especial - Educação do Indivíduo Especial) - Universidade Federal de São Carlos, São Carlos, 2003.

CABRERA, N.; BROOKS-GUNN, J.; MOORE, K.; WEST, J.; BOLLER, K.; TAMIS-LE MONDA, C. S. Bridging research and policy: Including fathers of young children in national studies. In: TAMIS-LE MONDA, C. S.; CABRERA, N. Handbook of father involvement: multidisciplinary perspectives. New Jersey: Lawrence Erlbaum Associates, 2002. p. 489-523, 2002.

CALDERA, Y. M. Paternal involvement and infant-father attachment: A Q-set study. Fathering: A Journal of Theory, Research and Practice, v. 2, p. 191-210, 2004.

CARPENTER, B. Sustaining the family: Meeting the needs of families of children with disabilities. British Journal of Special Education, v. 27, n. 3, p. 135-144, set. 2000.

CIA, F. Um programa para aprimorar envolvimento paterno: Impactos no desenvolvimento do filho. Tese (Doutorado em Educação Especial - Educação do Indivíduo Especial) - Universidade Federal de São Carlos, São Carlos, 2009.

; D’AFFONSECA, S. M.; BARHAM, E. J. A relação entre envolvimento paterno e desempenho acadêmico dos filhos. Paidéia Cadernos de Psicologia e Educação, Ribeirão Preto, v. 14, n. 29, p. 277-286, set/dez., 2004.

COLnAGO, N. A. S. Orientação para pais de crianças com Síndrome de Down: elaborando e testando um programa de intervenção. Tese (Doutorado em Psicologia) Universidade de São Paulo, Ribeirão Preto, 2000.

DA SILVA, N. C. B. Contexto familiar de crianças com Síndrome de Down: interação e envolvimento paterno e materno. Dissertação (Mestrado em Educação Especial - Educação do Indivíduo Especial) - Universidade Federal de São Carlos, São Carlos, 2007.

. Intervenção domiciliar e envolvimento paterno: efeitos em famílias de crianças com síndrome de Down. Tese (Doutorado em Educação Especial - Educação do Indivíduo Especial) - Universidade Federal de São Carlos, São Carlos, 2011.

; AIELLO, A. L. R. Análise descritiva do pai da criança com deficiência mental. Estudos de Psicologia, Campinas, v. 26, n. 4, dez. 2009.

; OLIVEIRA, A. S. S.; SÁS, R. M. Brincadeiras e jogos no ensino do indivíduo com deficiência mental. Educare, Montes Claros, v. 1, p. 20-33, 2008.

DESSEN, M. A. O efeito do observador em estudos longitudinais dentro do contexto familiar: relato de uma experiência. Psicologia: Teoria e Pesquisa, v. 12, p. 97-101, 1996.

DOHERTY, W. J.; ERICKSON, M. F.; LAROSSA, R. An intervention to increase father involvement and skills with infants during the transition to parenthood. Journal of Family Psychology, v. 20, n. 3, p. 438-447, 2006. 
EMMEL, M. L. G. Proposições sobre o significado do brincar no desenvolvimento infantil. In: MENDES, E. G.; ALMEIDA M. A.; WILLIAMS, L. C. A. Temas em Educação Especial: Avanços Recentes. São Carlos: EdUFSCar, p. 85-89, 2004.

ENGLE, P. L.; BREAUX, C. Father's involvement with children: Perspectives from developing countries. Social Policy Report: Society for Research in Child Development, v. 12, n. 1, p. 1-24, 1998.

FAGAN, J.; IGLESIAS, A. Father involvement program effects on fathers, father figures, and their Head Start children: A quasi-experimental study. Early Childhood Research Quarterly, v. 14, n. 2, p. 243-269, 1999.

FLOURI, E.; BUCHANAN, A. The role of father involvement in children's later mental health. Journal of Adolescence, v. 26, p. 63-78, 2003.

GALLAGHER, J. J. The family as a focus for intervention. In: MEISELS, S. J.; SHONKOFF, J. P. Handbook of early childhood intervention. Cambridge: Cambridge University Press, p. 540-559, 1990.

KUMPFER, K. L.; ALVARADO, R. Family-strengthening approaches for the prevention of youth problem behaviors. American Psychologist, v. 58, p. 457-465, 2003.

LAMB, M. E. The role of the father in child development. New York: Wiley, 1997.

MAGGIL-EVANS, J.; HARRISON, M. J.; REMPEL, G.; SLATER, L. Interventions with father of young children: Systematic literature review. Journal of Advanced Nursing, v. 55, n. 2, p. 248-264, 2006.

McALLISTER, C. L.; WILSON, P. C.; BURTON, J. From Sports Fans to Nurturers: An Early Head Start Program's Evolution Toward Father Involvement. Fathering: A Journal of Theory, Research, and Practice About Men as Fathers, v. 2, n. 1, p. 31-59, 2004.

McBRIDE, B. A. The effects of a parent education/play group program on father involvement in child rearing. Family Relations, v. 39, p. 250-256, 1990.

MINCY, R. B.; POUNCY, H. W. The responsible fatherhood field: Evolution and goals. In: TAMIS-LE MONDA, C. S.; CABRERA N. Handbook of father involvement: Multidisciplinary perspectives, New Jersey: Lawrence Erlbaum Associates, p. 555-597, 2002.

NUNES, C. C.; DA SILVA, N. C. B.; AIELLO, A. L. R. As contribuições do papel do pai e do irmão do indivíduo com necessidades especiais na visão sistêmica da família. Psicologia: Teoria e Pesquisa, Brasília, v. 24, n. 1, mar. 2008.

OMOTE, S. Conclusões do grupo de trabalho (GT3): A família do deficiente. In: GOYOS, C.; ALMEIDA, M. A.; DE SOUZA, D. Temas em Educação Especial. 3. São Carlos: Editora da UFSCar, p. 516-525, 1996.

PELCHAT, D.; LEFEBVRE, H.; LEVERT, M. J. Gender differences and similarities in the experience of parenting a child with a health problem: Current state of knowledge. Journal of Child Health Care, v. 11, n. 2, p. 112-131, 2007. 
PEREIRA-SILVA, N. L.; DESSEN, M. A. Intervenção precoce e família: contribuições do modelo bioecológico de Bronfenbrenner. In: DESSEN, M. A.; JUNIOR, A. L. C. (Ed.). A ciência do desenvolvimento humano: tendências atuais e perspectivas futuras. Porto Alegre: Artmed, p. 152-167, 2005.

QUESENBERRY, A.; OSTROSKY, M. M.; CORSO, R. Skilled and knowledgeable caregivers: The role of fathers in supporting young children's development. Young Exceptional Children, v. 10, n. 4, p. 11-19, 2007.

RAIKES, H.; BOLLER, K.; VAN KAMMEN, W.; SUMMERS, J. A.; RAIKES, A.; LAIBLE, D.; WILCOX, B.; ONTAI, L.; CHRISTENSEN, L. Father involvement in Early Head Start Programs: A practitioners study. 2002. Disponível em: <http://ccfl. unl.edu/projects_outreach/projects/previous/pdf/Final_Father_Involvement_Report. pdf>. Acesso em: 12/07/2009.

SPIKER, D.; HOPMANN, M. R. The effectiveness of early intervention for children with Down Syndrome. In: GURALNICK; M. J. Effectiveness of early intervention. Baltimore: Paul Brookes, p. 271-305, 1997.

TURBIVILLE, V. P.; MARQUIS, J. G. Father participation in early education programs. Topics in Early Childhood Special Education, v. 21, n. 4, p. 223-231, 2001.

WILliamS, L. C. A.; AIEllo, A. L. R. O Inventário Portage Operacionalizado: intervenção com famílias. São Paulo: Memnon, 2001.

. Empoderamento de famílias: o que vem a ser e como medir. In: MEN-

DES, E. G.; ALMEIDA M. A.; WILLIAMS, L. C. A. Temas em Educação Especial: avanços recentes. São Carlos: Editora da UFSCar, p.197-202, 2004.

Texto recebido em 05 de setembro de 2011.

Texto aprovado em 28 de outubro de 2011. 